\title{
ANALYSIS OF CURRENT REDISTRIBUTION IN A CICC UNDER TRANSIENT HEAT PULSES
}

\author{
L. Bottura ${ }^{1}$, P. Bruzzone ${ }^{2}$, and C. Marinucci ${ }^{2}$
}

We have performed experiments and simulations of the current distribution process in a CICC with the aim to understand better the coupled thermal, hydraulic and electric process that leads to a stable or unstable transient cable behaviour. The cable, wound from 128 $\mathrm{Nb3Sn}$ and pure copper strands, has been tested in the SULTAN facility. A resistive heater, glued on the jacket of the conductor, has been used to start the transient, and the response has been monitored with arrays of Hall plates. In this paper we report the results of simulations, especially the computed Hall signals, and compare them to the experimental data. Based on the experimental results and their interpretation we postulate that large temperature gradients must develop in the helium stream in the cable cross sections during the transient heat pulse.

1 CERN, Accelerator Technology Department, Geneva, Switzerland

2 Fusion Technology Division, EPFL/CRP, Villigen, Switzerland

\author{
Presented at the 2003 Cryogenic Engineering Conference and International Cryogenic Materials Conference \\ CEC/ICMC 2003 \\ 22-26 September 2003, Anchorage, Alaska
}

Administrative Secretariat

AT Division

CERN

$\mathrm{CH}$ - 1211 Geneva 23
Geneva, Switzerland

29 January 2004 


\title{
ANALYSIS OF CURRENT REDISTRIBUTION IN A CICC UNDER TRANSIENT HEAT PULSES
}

\author{
L. Bottura ${ }^{\mathrm{a}}, \mathrm{P}$. Bruzzone $^{\mathrm{b}}$, and C. Marinucci ${ }^{\mathrm{b}}$ \\ ${ }^{\mathrm{a}}$ AT Division, CERN \\ CH-1211, Geneva, Switzerland \\ ${ }^{\mathrm{b}}$ Fusion Technology Division, EPFL/CRPP \\ CH-5232, Villigen, Switzerland
}

\begin{abstract}
We have performed experiments and simulations of the current distribution process in a CICC with the aim to understand better the coupled thermal, hydraulic and electric process that leads to a stable or unstable transient cable behaviour. The cable, wound from $128 \mathrm{Nb}_{3} \mathrm{Sn}$ and pure copper strands, has been tested in the SULTAN facility. A resistive heater, glued on the jacket of the conductor, has been used to start the transient, and the response has been monitored with arrays of Hall plates. In this paper we report the results of simulations, especially the computed Hall signals, and compare them to the experimental data. Based on the experimental results and their interpretation we postulate that large temperature gradients must develop in the helium stream in the cable cross sections during the transient heat pulse.
\end{abstract}

\section{INTRODUCTION}

Current distribution and redistribution is known to have an impact on the stability properties of superconducting cables used for fusion [1], and on the quality of the field homogeneity in superconducting accelerator magnets [2]. The amount of current imbalance, the mechanism by which current distribute among the superconducting strands in a cable, and the consequences of this process are matters that are hardly controllable during design and construction of the cable. Research in this field is nonetheless important to improve our understanding of the fundamental aspects of the current distribution mechanisms, so that consequences can be anticipated based on scaling of experimental results and analytical modelling.

Here we analyse the details of a transient stability experiment performed in the SULTAN facility on a mid-size $\mathrm{Nb}_{3} \mathrm{Sn}$ Cable-in-Conduit Conductor [3-5]. The experiment was mainly aimed at verifying that the current can be shared among strands following a 
TABLE 1. Main Characteristics and Operating Conditions of the Nb3Sn Sample

\begin{tabular}{llllllc}
\hline SC Strand Diameter & $(\mathrm{mm})$ & 0.82 & & Operating current & $(\mathrm{A})$ & 14300 \\
SC Strand Cu:non-Cu & $(-)$ & 1 & & Operating field & $(\mathrm{T})$ & 10 \\
Cu Strand Diameter & $(\mathrm{mm})$ & 1.1 & & Inlet temperature & $(\mathrm{K})$ & 4.6 \\
Number of SC strands & $(-)$ & 112 & & Inlet pressure & $(\mathrm{bar})$ & 10 \\
Number of Cu strands & $(-)$ & 16 & & Mass flow & $(\mathrm{g} / \mathrm{s})$ & 3 \\
Non-Cu cross section & $\left(\mathrm{mm}^{2}\right)$ & 29.57 & & & \\
Cu cross section & $\left(\mathrm{mm}^{2}\right)$ & 44.77 & & & \\
Void Fraction & $(\%)$ & 37.2 & & & \\
Strand Wetted Perimeter & $(\mathrm{mm})$ & 343 & & \\
Cable Space Diameter & $(\mathrm{mm})$ & 12.54 & &
\end{tabular}

localised normal transition. The cable, whose cross section is shown in FIG 1, consists of $112,0.82 \mathrm{~mm}$ diameter, $\mathrm{Cr}$-plated $\mathrm{Nb}_{3} \mathrm{Sn}$ strands and of $16,1.1 \mathrm{~mm}$ pure $\mathrm{Cu}$ strands cabled in a $(1+7) \times 4 \times 4$ pattern. The main conductor characteristics are summarised in TABLE 1 , while details can be found elsewhere [4-5]. The sample, an hairpin of approximately $3 \mathrm{~m}$ length (6 m total cable length) also shown schematically in FIG 1, was placed in the SULTAN split solenoid and extensively tested to determine its DC and AC characteristics [5]. A particularity of relevance is that the sample showed a broadening of the resistive transition after several thousands powering cycles, with a power-law index $n$ as low as 6 at $10 \mathrm{~T}, 4.6 \mathrm{~K}[6]$.

Current redistribution in a localised normal zone was induced firing a short resistive heater, centered in the high field region of the sample (10 T over approximately $0.6 \mathrm{~m})$. The heater was glued on the jacket and provided a power input localised both in length $(2 \mathrm{~cm}$ nominal heating length) as well as in the conductor cross section (6 mm width). The current redistribution process was monitored by sets of Hall plates measuring the changes in the self-field of the sample. The Hall plates were arranged in rings, and oriented parallel to the background field so to partially compensate it and increase the dynamic for the measurement of the self field of the sample. Two rings are considered in the analysis below, namely ring $\mathrm{H} 2$, located $15 \mathrm{~mm}$ from the heater, downstream in the direction of the helium flow, and ring $\mathrm{H} 3$, placed at approximately $0.5 \mathrm{~m}$ from the heater, upstream in the direction of the helium flow.

The experiment analysed here is a run at $14.2 \mathrm{kA}, 4.6 \mathrm{~K}$ and $10 \mathrm{~T}$ (run BDI190722) where the resistive heater was powered in a short pulse $(0.85 \mathrm{~s}$ duration) with an electric power of $20 \mathrm{~W}$. The sample underwent a normal transition followed by recovery of the equilibrium state after, typically, $2 \mathrm{~s}$. The normal transition was recorded by resistive voltage in the high field region, as well as by signals at the Hall plate rings, shown in

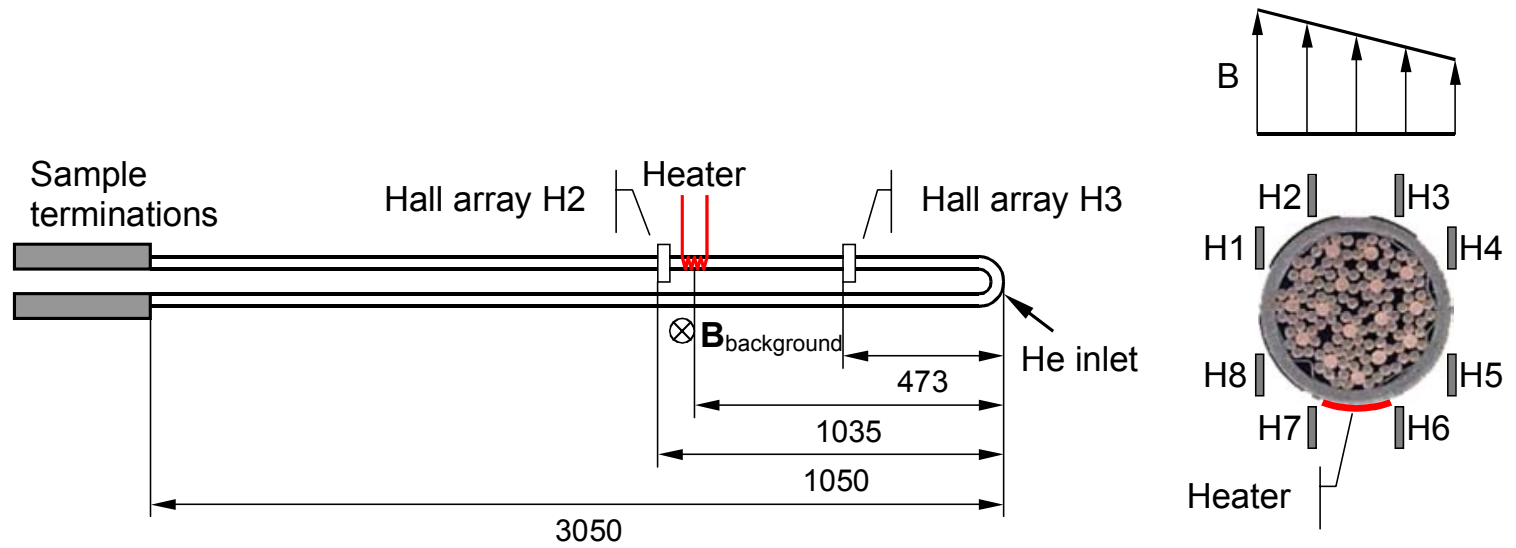

FIGURE 1. Schematics of the hairpin sample, reporting the longitudinal location of the heater and of the Hall plate rings, and cross section of the conductor, showing the location of the eight Hall plates in each ring, and of the resistive heater relative to the field profile across the cable. 

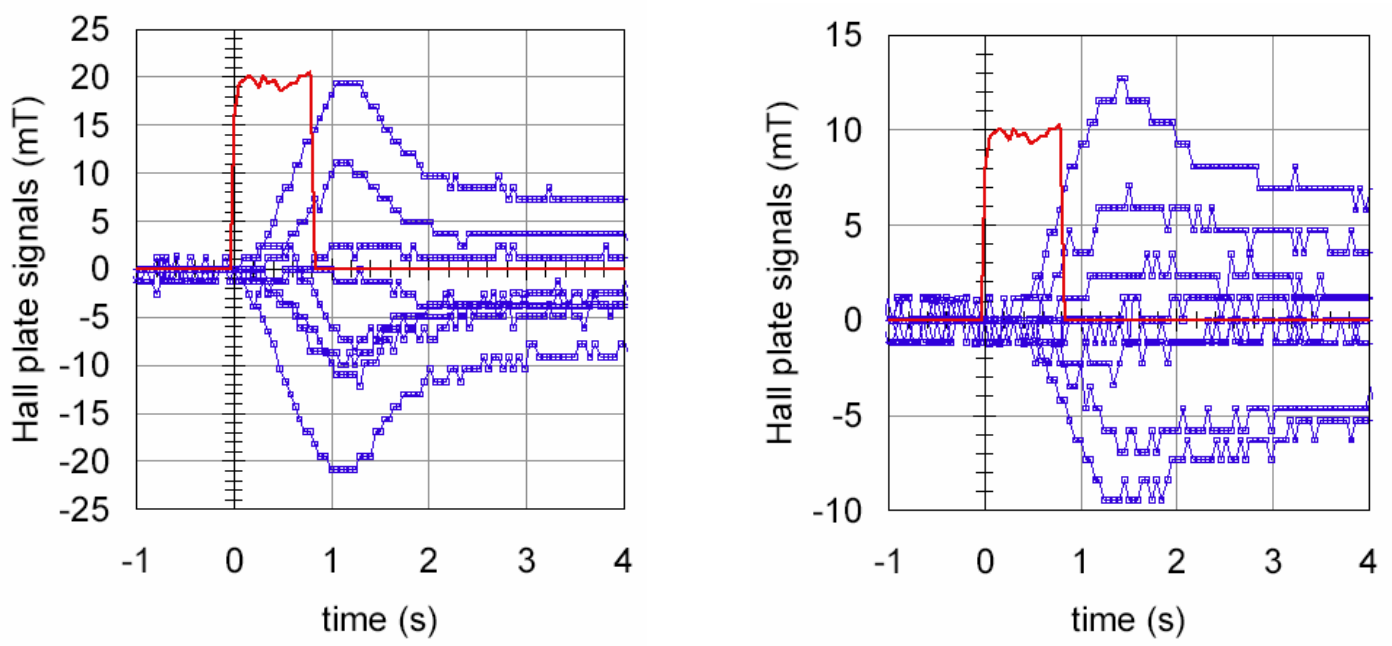

FIGURE 2. Signals at the Hall plates of rings $\mathrm{H} 2$ (left, symbols) and $\mathrm{H} 3$ (right, symbols) during a heating pulse with $20 \mathrm{~W}$ electric power and $0.85 \mathrm{~s}$ duration (the heater waveform is reported as a solid line). The initial offset has been removed from the Hall plate signals to evidence the changes in the self field.

FIG 2. The signals of FIG 2 show a strong response of the H2 Hall plates, sensing the current displacement in the cable just below the heater, with peak field changes of $20 \mathrm{mT}$. The onset of the response at the $\mathrm{H} 3$ ring is delayed by approximately $0.3 \mathrm{~s}$, and its magnitude is sensibly smaller, about $10 \mathrm{mT}$. As this Hall plate ring is upstream in the direction of the helium flow, and a temperature sensor located in the vicinity shows essentially no response, the change in the cable self field at the $\mathrm{H} 3$ location can be attributed to the diffusion of current.

\section{ANALYSIS OF THE CURRENT REDISTRIBUTION}

We have used the field measured by the Hall plates, reported in FIG 2, to reconstruct the current distribution inside the cable. The general free boundary inverse electromagnetic problem, consisting in finding the current distribution inside the cable space that gives the measured magnetic field, is known to be ill-conditioned [7]. In practice this problem cannot be solved with a reasonable degree of confidence for quantitative analysis of experimental data. To regularise the problem, and make the solution possible, the cable space is subdivided in a limited set of $m$ current carrying elements. The field generated by each element at the location of the $n$ sensors can be computed by standard techniques. The result of this calculation is a set of linear equations:

$$
\mathbf{G I}=\mathbf{B}
$$

where $\mathbf{I}$ is the array of the $m$ currents in the current elements, $\mathbf{B}$ is the array of the magnetic field measured by the $n$ sensors, and $\mathbf{G}$ is the $n \times m$ influence matrix relating the two. The matrix $\mathbf{G}$ is not necessarily square, and it could be singular if the location of the current elements in the cable space is arbitrarily chosen. To obtain the value of $\mathbf{I}$ the standard practice is to perform a pseudo-inversion by Singular Value Decomposition [8], minimising the $\mathrm{L}_{2}$ norm of the error on the field, i.e. $\| \mathbf{B}$ - GI $\|$, as well as the norm of the amplitude of the currents $\|\mathbf{I}\|$.

For our analysis of the 8 signals of each Hall plate ring we have placed the current elements, 8 filaments, on a circle of radius equal to half the cable space radius. The reconstructed current changes in each filament are plotted in polar coordinates in FIG 3 at four instants during the evolution. The amount of current that redistributes in the cross 

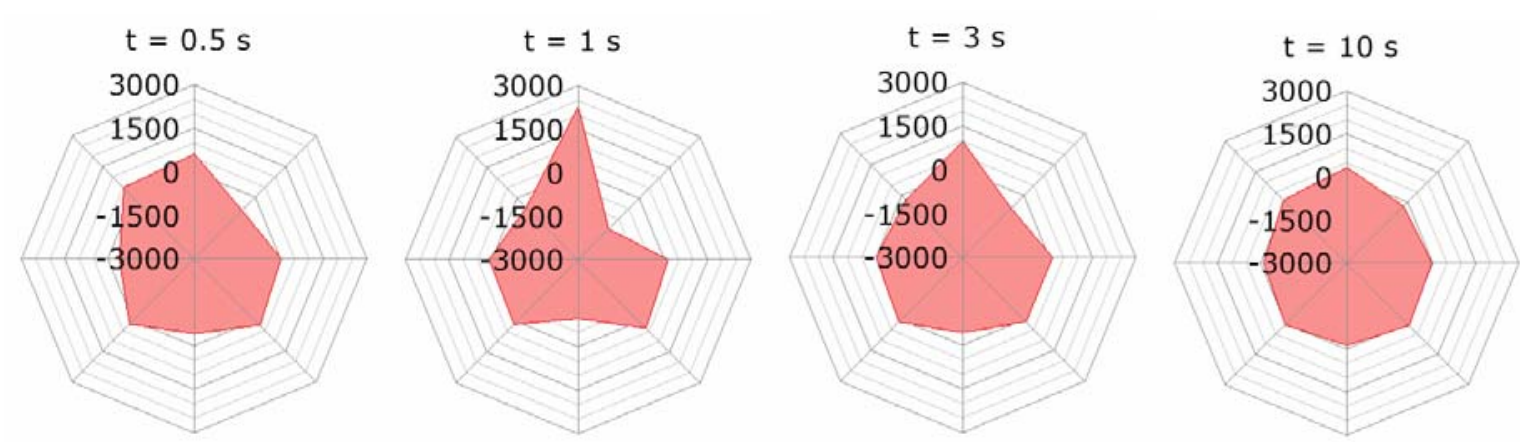

FIGURE 3. Reconstructed plot of the current changes as a function of position in the cable.

section has a peak of the order of $2 \mathrm{kA}$ at the end of the heating pulse. The initial distribution is recovered after a few $\mathrm{s}$ from the end of the pulse. In normal operating conditions, and with a perfect current sharing among strands, the current carried by each strand would be 115 A. A current displacement as shown in FIG 3 would thus correspond to $15 \%$ of the strands fully normal conducting, so that we see clearly that the transient involves a large fraction of the cable.

The time evolution of the current diffusion gives additional important information on the electrical cable characteristics. As discussed at length in [9], the decay of the current induced by a fast voltage pulse in a multistrand cable follows a time evolution that can be well approximated by a series of exponentials. Assuming that the self field change has the same dynamics as the current redistribution, we have obtained the longest decay time constant fitting an exponential function to the Hall plate signals at times sufficiently long after the end of the pulse $(0.5 \mathrm{~s})$. The result of the fit is a range of decay time constants from 2 to $2.5 \mathrm{~s}$. In this analysis it is important to remove the final offset of the cable self field that corresponds to a change in current distribution frozen in the cable after the pulse. This change, attributed to current rearrangement at the joints, has in practice very long time constants.

\section{SIMULATION OF THE EXPERIMENTAL RUN}

\section{Description of the Model}

To understand the physics underlying the response of the cable to the heating pulse we have simulated the transient using a 1-D numerical model that allows an arbitrary subdivision of the cable cross section in components with uniform properties and the simultaneous solution of the coupled electrical, thermal and hydro-dynamic problem [10]. We have modelled the current distribution using four equal super-strands corresponding to the last-but-one cabling stage. The choice of this level of approximation is justified by the previous considerations on the amount of cable involved in the current transient, which is a sizeable fraction (more than half) of the last-but-one cabling stage. The electrical components are coupled inductively (through mutual inductance) and resistively (through interstrand conductance). The temperature field in the cable was modelled subdividing the cable in a similar manner, i.e. four parallel components with the size of the last-but-one cabling stage. In addition, to model properly the heating process through the conductor jacket, we have considered the jacket as split in two independent thermal components. The first, smaller element simulates the portion of the jacket below the heater, while the second element models the rest of the jacket. The thermal components are coupled by thermal resistances that simulate the heat transfer at the contact surfaces. Finally, as discussed later, it is important to consider that also the helium state is not uniform in the cable cross 
Electric components

(super-strands)

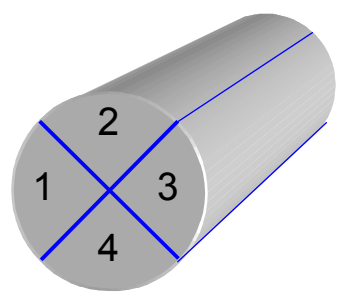

Thermal components

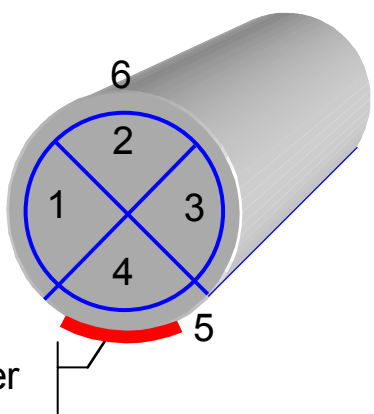

Hydraulic components (helium channe/s)

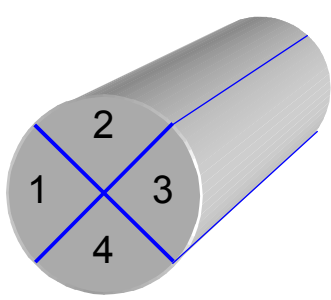

FIGURE 4. Schematic representation of the model used for the analysis. The cable cross section is subdivided in electric, thermal and hydraulic components, whose state is defined by current, temperature and flow state variables respectively. Thermal component 5 is the portion of the jacket below the heater, thermal component 6 is the rest of the jacket.

section. For this reason we have modelled the helium flow as a parallel of four independent channels, thermally coupled to each thermal component as well as among themselves through both heat and mass transport. A schematic representation of the components for each domain is shown in FIG 4. The complete sample model is obtained extruding the cross section of FIG 4 in the direction of the cable length. Further details on the model, and in particular the governing equations in each domain (current, temperature, helium state and flow) and the solution methods can be found elsewhere [10].

The choice of the model input data is obviously the most critical step in the preparation of the simulation. Apart for the geometrical quantities (cross sections, wetted perimeter, hydraulic diameter, inductances) that can be derived in a straightforward manner from the cable data, we have found the results discussed below to be most sensitive to the heating waveform, the interstrand conductance per unit length and the thermal resistances that couple thermal and hydraulic components.

The waveform of the heat deposition from the heater to the jacket (and eventually to the adjacent strands and helium) is not only determined by the electrical power supplied to the heater strip, but also by the heater heat capacity and the thermal resistances that tend to delay heat flow to the cable. This effect can be modelled to the first order as an exponential front on the onset of the electrical pulse and an exponential decay after the end of the electrical pulse. The time constant of the exponential can be estimated in a simple-minded model as the product of the heat capacity of the heater (in $[\mathrm{J} / \mathrm{K} \mathrm{m}]$ ) and thermal resistance from the heater to the jacket (in $[\mathrm{K} \mathrm{m} / \mathrm{W}]$ ). Both the heat capacity and the thermal resistance are strong function of temperature in the range spanned during the heating transient ( $4.5 \mathrm{~K}$ at the beginning to approximately $40 \mathrm{~K}$ at the end of the pulse), therefore we have used different time constants for the onset and the decay exponentials. The estimated values are $50 \mathrm{~ms}$ for the heating onset and $400 \mathrm{~ms}$ for the heating decay.

We have deduced a range of realistic values of the interstrand conductance per unit length $g$ using the analytical result of [9] for the time constant of free current decay in an $\mathrm{N}$ strand cable:

$$
\tau=N(l-m) g\left(\frac{L}{\pi}\right)^{2}
$$

where $(l-m)$ is the inductance of the return line formed by two last-but-one stages, ranging from 0.12 to $0.19 \mu \mathrm{H} / \mathrm{m}$, and $L$ is the cable length between joints, $3 \mathrm{~m}$ in our case (we consider the sample to be symmetric around its mid-point). Taking values of $\tau$ in the range 
2 to $2.5 \mathrm{~s}$, as derived from the fit of the Hall plate signals, we obtain a range of $g$ from 2.8 MS/m to $4.8 \mathrm{MS} / \mathrm{m}$. We have performed simulations around the central value, i.e. $3.8 \mathrm{MS} / \mathrm{m}$. The range above happens to be consistent with the values obtained from direct measurement of interstrand resistance on short samples of the same conductor [4], from 300 to $600 \mathrm{n} \Omega \mathrm{m}$ that corresponds to values of $g$ from $1.6 \mathrm{MS} / \mathrm{m}$ to $3.3 \mathrm{MS} / \mathrm{m}$. A necessary caveat is however that while the short sample measurements refer to virgin conditions, the tests discussed here are for a sample that underwent intense cyclic electromagnetic loading, so that the agreement of the range of values obtained for $g$ could be accidental.

The thermal coupling among the different components (cable, jacket and helium stream) is definitely the most uncertain matter. The thermal coupling of cable and helium is through heat transfer through the turbulent boundary layer at the wetted perimeters of the strands. Furthermore, we have assumed that the jacket is coupled to the helium as well as to the cable through a thermal resistance $R_{S S}$ per unit length dominated by the conductivity of the jacket itself (estimated at $R_{S S}=0.25 \mathrm{~K} \mathrm{~m} / \mathrm{W}$ ). In contrast, no direct thermal coupling was assumed among the four thermal components modelling the cable (i.e. last-but-one to last-but-one cabling stage). Finally, the helium channels were coupled through turbulent heat transfer at a fictitious wetted perimeter equal to the radius of the cable space, i.e. as schematically represented in FIG 4.

\section{Results of the Simulation and Comparison to Measured Data}

The main result of the simulation is the diffusion of current caused by the local transition of the superconductor. This process is shown in FIG 5, where we have reported snapshots of the current profile in the four electric components at selected times during and after the heating pulse. The largest current variations are obtained, as expected, close to the heater, i.e. at around $1 \mathrm{~m}$ from the helium inlet (see the schematics of FIG 1). The current diffusion wave reaches the top joint, at $3 \mathrm{~m}$ in FIG 5, right after $0.5 \mathrm{~s}$ from the beginning of
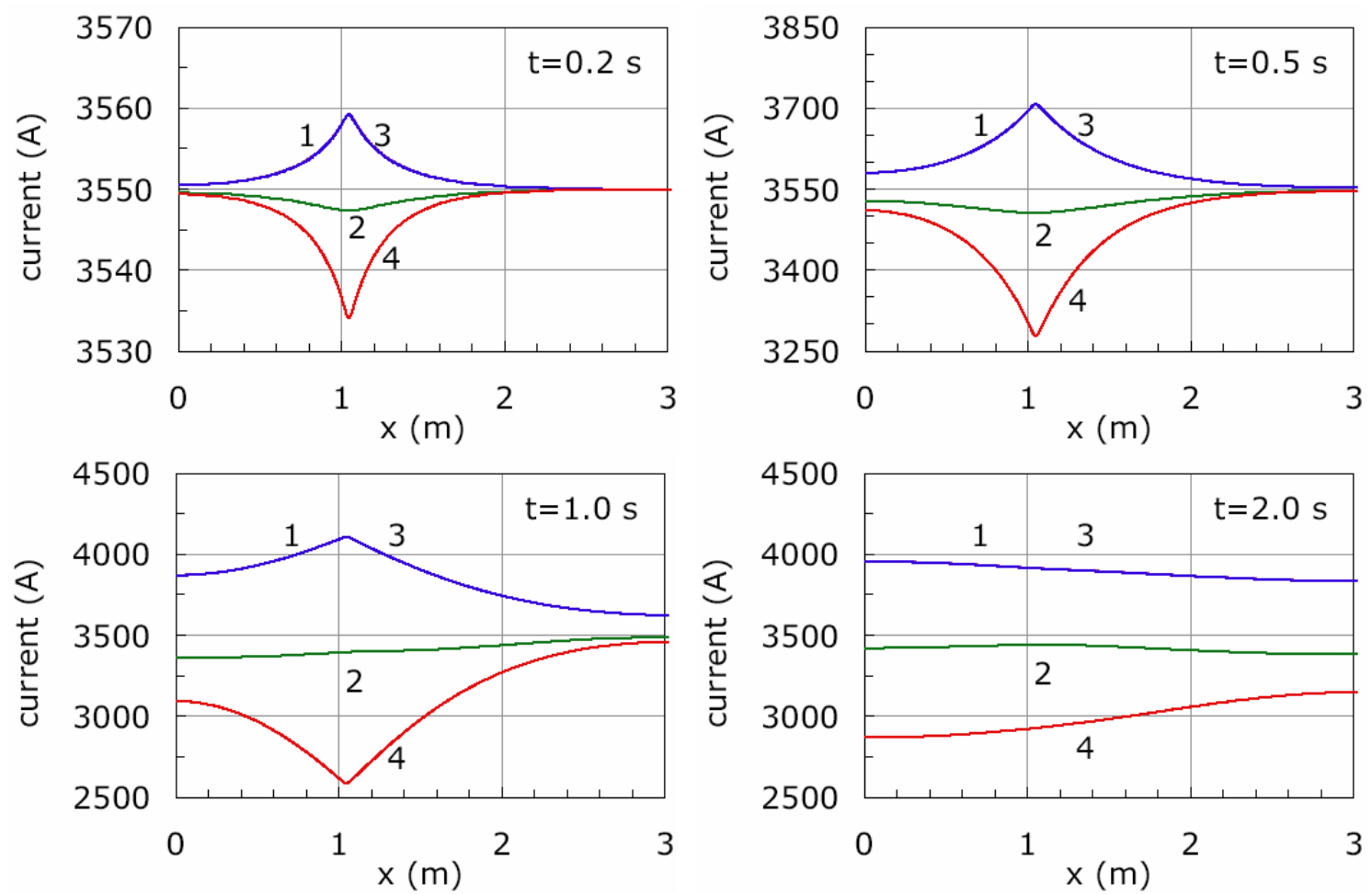

FIGURE 5. Profile of current in the four electric components at selected times during and after the heating pulse. The current in the components 1 and 3 is nearly identical. Note the different scales used for the y-axis to increase readibility. 
the pulse, which is consistent with the observation in [3] of a longitudinal current distribution velocity of $4 \pm 0.5 \mathrm{~m} / \mathrm{s}$. This also implies that tests with a sample of this length are influenced by the boundary conditions imposed by the sample terminations and that care should be exerted when extrapolating the results of short samples to a coil.

The time evolution of the current was used to compute the field at the Hall plates in the $\mathrm{H} 2$ and $\mathrm{H} 3$ rings using the linear equation (1). The result of this calculation is compared to the data recorded during the experiment in FIG 6. The agreement between experiment and simulation is satisfactory: the amplitude of the signals as well as the dynamics are well matched. The maximum errors are of the order of $20 \%$ of the measured peak field, which we attribute to the simplifications taken in the model.

Any variation of the model input parameters from the nominal choices outlined previously results in increasing deviations from this satisfactory match. One striking example is shown in FIG 7 where we show the effect of making the assumption that the helium stream in the cable space has a uniform temperature (i.e. we take a single helium channel in the model). The helium in this case acts as an artificially large buffer and tends to make the conductor less responsive to temperature excursions. The resulting current
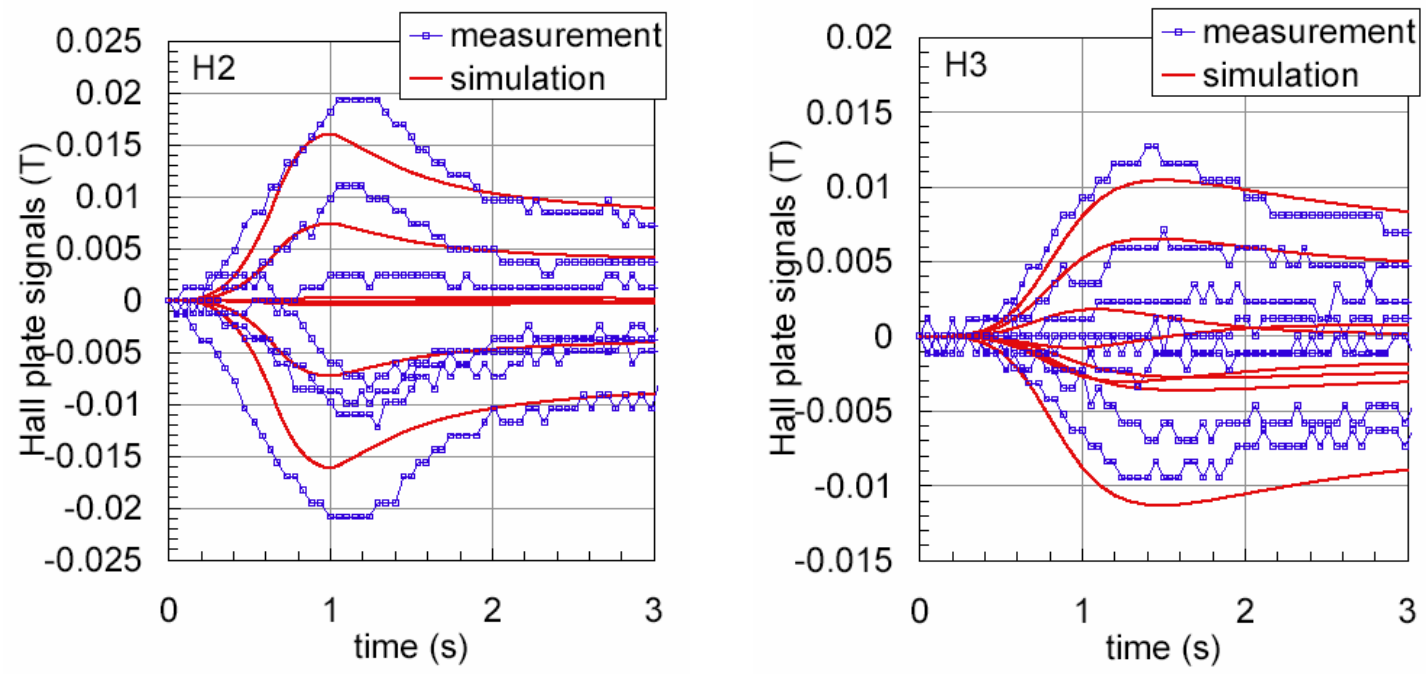

FIGURE 6. Comparison of measured and simulated Hall plate signals at the rings $\mathrm{H} 2$ and $\mathrm{H} 3$. The simulations were performed with nominal input parameters as discussed in the text.
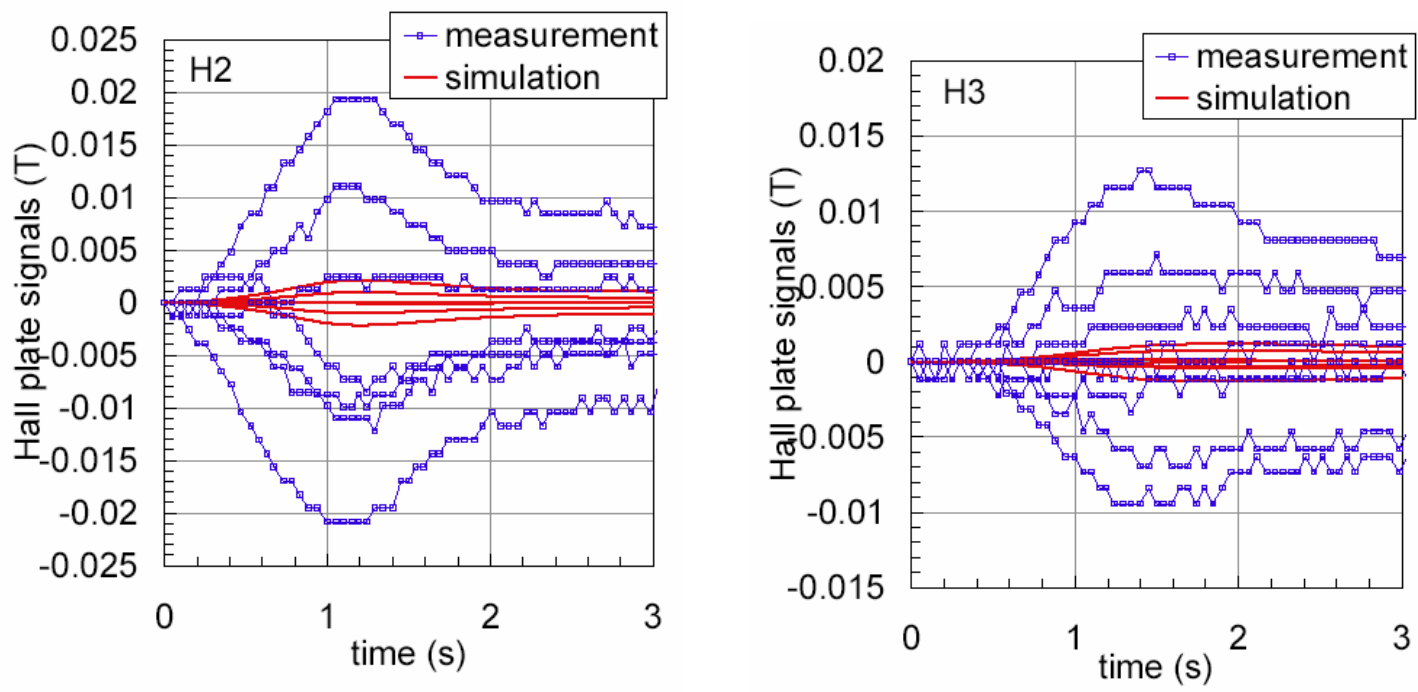

FIGURE 7. Effect of the model of the helium flow on the simulated Hall plate signals at the rings $\mathrm{H} 2$ and $\mathrm{H} 3$. The measred signals are compared to the result of the simulation in the case of a single helium channel with cross section equal to the total helium amount in the cable space. 
distribution process is much reduced and deviates largely from the measurement. This result also points at the fact that for localised heating the temperature gradient inside the cable space is large. The values obtained from the simulation are in excess of $1.5 \mathrm{~K}$. Similar results were observed experimentally on a large CICC tested in comparable conditions [11], and we thus believe that the temperature gradients from the simulation are of sound physical nature.

\section{CONCLUSIONS}

We have shown that the current redistribution properties of a small-size CICC under localised heat input can be explained to a satisfactory and self-consistent level once temperature gradients in the cross section of the cable are postulatd and taken into account. Although the presence of temperature and flow gradients inside a CICC is not a new finding (see [12], [13] and [11] for the various aspects of flow and temperature nonuniformities) these effects are generally neglected in the design and analysis of cables and coils. According to our results, however, it appears that a better understanding of both is of paramount importance to grasp to the full extent the basics of coupled electrodynamics and thermo-hydraulics of a CICC.

From a practical point of view, a level of approximation as taken here, i.e. modelling the last-but-one stage of the cable, appears to be sufficient to describe the transients of interest. It is however still open whether this level of approximation is applicable to the analysis of larger cables and other operating conditions than considered here. This question can only be resolved by further detailed analysis of experiments.

\section{REFERENCES}

1. Mitchell, N., "Analysis of non-Uniform Current Distribution Effects in Multistage Cable-in-Conduit Conductors", Cryogenics 39, pp 539-556 (1999).

2. Bottura, L., Walckiers, L. and Wolf, R., "Field Errors Decay and "Snap-Back" in LHC Model Dipoles", IEEE Trans. Appl Sup. 7, 1997, pp 602-605.

3. Bruzzone, P., Fuchs, A., Stepanov, B. and Vecsey, G., "Transient Stability Results for Nb3Sn Cable-inConduit Conductors", IEEE Trans. Appl. Sup. 12, 2002, pp 512-515.

4. Bruzzone, P., Shikov, A., Vorobieva, A., Sytnikov, V., Nijhuis, A. and Specking, W., "Characterization Tests of the Nb3Sn Cable-in-Conduit for SeCRETS”, IEEE Trans. Appl. Sup. 10, 2000, pp 1086-1089.

5. Bruzzone, P., Fuchs, A.M., Stepanov, B., Vecsey, G. and Zapretelina, E., "Test Results of SeCRETS, a Stability Experiment About Segregated Copper in CICC", IEEE Trans. Appl. Sup. 11, 2001, pp 20182021.

6. Bruzzone, P., Fuchs, A.M., Stepanov, B. and Vecsey, G., "Performance Evolution of Nb3Sn Cable-inConduit Conductors under Cyclic Load”, IEEE Trans. Appl. Sup. 12, 2002, pp 516-519.

7 Ilyin, Y.A. and Nijhuis, A., "Case Studies in Reconstruction Efficiency of Current Distribution in CICC's by Self Field Measurements”, IEEE Trans. Appl. Sup. 13, 2003, pp 1748-1751.

8 Press, W., Flannery, B., Teukolsky, S. and Vetterling, W. T., Numerical Recipes, Cambridge University Press, 1990.

9 Bottura, L., Breschi, M. and Fabbri, M., “An Analytical Benchmark for the Calculation of Current Distributionin Superconducting Cables”, Cryogenics 43, pp 241-248 (2003).

10 Bottura, L., Rosso, C. and Breschi, M., “A General Model for Thermal, Hydraulic, and Electric Analysis of Superconducting Cables”, Cryogenics 40, pp 617-626 (2000).

11. Bruzzone, P., Fuchs, A., Vecsey, G. and Zapretelina, E., "Test Results for the High Field Conductor of the ITER Central Solenoid Model Coil”, Adv. Cryo. Eng. 45, 2000, pp 729-736.

12. Amano, T., et al., "Flow Visualization of Coolant in Cable-in-Conduit Conductor", IEEE Trans. Mag. 27, 1991, pp 2112-2115.

13. Daugherty, M.A., "Helium Hydrodynamics of Internally Cooled Cabled Superconductors", Ph.D. Thesis, University of Wisconsin-Madison, 1991. 